\title{
NOTAS
}

\section{LA NOCIÓN DE TRADICIÓN VERBAL Y SU VALOR PARA LA LINGÜÍSTICA HISTÓRICA}

La construcción de conceptos forma parte muy importante del desarrollo de una ciencia. Mientras que para el común de la gente lo que destaca de las ciencias son sus teorías y sus resultados como productos terminados, ni los largos procesos de construcción de instrumentos de observación, medida y experimentación, ni los complicados rejuegos que llevan a la construcción de conceptos de análisis y de teorización son objeto de interés e incluso se suelen soslayar por completo.

La lingüística estructural dotó a la ciencia de un complejo arsenal de conceptos, cuya característica última es que se trata de conceptos descriptivos de fenómenos verbales considerados como producto, o dicho con Humboldt, Bühler y Coseriu, como ergon ${ }^{1}$; y contra lo que se podría suponer, la lingüística chomskyana, que abrió una perspectiva dinámica de los fenómenos lingüísticos, con base en sus conceptos centrales de competencia, regla de transformación y recursividad dio lugar sólo a una conceptualización formalista, restringida a los hipotéticos sistemas de las lenguas, que no llegó a cuajar en una teoría completa, orgánica y empíricamente verificable, y mucho menos parece haber considerado la evolución de las lenguas, ni como problema diacrónico, ni como problema histórico.

Desde hace varios años ha habido múltiples esfuerzos por superar la concepción estructuralista, heredada de la enseñanza de Ferdinand de Saussure, Louis Hjelmslev y, paradójicamente, Eugenio

1 Wilhelm von Humboldt, Über die Verschiedenheit des menschlichen Sprachbaues und ihren Einfluss auf die geistige Entwicklung des Menschengeschlechts, en Schriften zur Sprachphilosophie, t. 3, Werke, 5. Unveränderte Auflage, J.G. Cotta'sche Buchhandlung, Stuttgart, 1979; Karl Bühler, Teoría del lenguaje, $2^{\mathrm{a}}$ ed., trad. J. Marías, Revista de Occidente, Madrid, 1967, I, § 4,1, y E. CoseriU, "Sistema, norma y habla", § 3, en Teoría del lenguaje y lingüística general. Cinco estudios, $3^{\mathrm{a}}$ ed. rev. y corr., Gredos, Madrid, 1973 y en varios otros estudios suyos. 
Coseriu $^{2}$, en dirección a la pragmática, a la lingüística del texto o del discurso y a la lingüística histórica.

Ahora bien, entre los conceptos científicos los hay, al menos, de dos clases: descriptivos de un estado de lengua, derivados de la epistemología positivista descendiente de Saussure y de Bloomfield, que originan los métodos de descripción y de clasificación de los fenómenos verbales observados, y teóricos, que resultan, en el caso considerado, de esa misma epistemología y sirven para explicar y entender las observaciones hechas; lamentablemente, muchas veces se elaboran conceptos "teóricos" que no provienen del análisis y el razonamiento de los fenómenos observados, sino que se difunden a priori por una mera mecánica de sistematicidad y de clasificación, como si correspondieran a la realidad fenoménica, a la que terminan forzando; pues hay que tomar en cuenta que, ante la extrema complejidad de los fenómenos del ser humano, en cuanto a su existencia espiritual, las teorías no pueden ser positivas, sino interpretativas, y sus métodos, hermenéuticos; por algo Kant consideró las humanidades (incluidas, por supuesto, las actuales "ciencias sociales") ciencias idiográficas y no nomotéticas, como las naturales.

En este artículo me ocuparé de los conceptos propuestos por Coseriu, de tradición verbaly de tradición discursiva $a^{3}$ en relación con la lingüística histórica, que han dado lugar a valiosos desarrollos posteriores, mediante los cuales la lingüística supera la epistemología positivista, llámese estructuralismo, chomskyanismo o sociolingüística funcionalista; son conceptos teóricos, sustentados en la observación de la historia de lenguas como el español, que cuentan con abundantes datos desde hace más de un milenio; pero en vez de limitarme a seguir su desarrollo, con una bibliografía tan extensa como se puede leer en el artículo que acabo de citar de Araceli López Serena, me propongo elaborarlos

${ }^{2}$ Digo paradójicamente, porque siempre me ha parecido que los conceptos centrales de Coseriu: sistema, norma y habla, lengua funcionaly los de su semántica son claramente estructuralistas, a pesar de las perspectivas no estructuralistas que abre su teoría del lenguaje, más propiamente humboldtiana, manifiesta, precisamente, en sus concepciones acerca del hablar, del saber hablary de la lengua histórica. Véase al respecto mi libro El concepto de norma en lingüistica, El Colegio de México, México, 1976.

${ }^{3}$ La pareja en lengua alemana de Sprechtraditionen y Sprachtraditionen se puede interpretar en español mediante tradiciones del hablar, tradiciones verbales y tradiciones lingüisticas. Las tradiciones discursivas forman parte de las primeras, y precisamente al respecto hay varias versiones entre los seguidores de estas ideas, sobre todo a partir de la obra de Brigitte Schlieben-Lange, Traditionen des Sprechens, Kohlhammer, Stuttgart, 1983, posteriormente desarrolladas por Peter Koch, Wulf Oesterreicher, Johannes Kabatek y Daniel Jacob. Véanse al respecto RaYmund Wilhelm, "Diskurstraditionen", en Language typology and language universals. An international Handbook, eds. M. Haspelmath et al., W. de Gruyter Verlag, Berlin, 2001, t. 1, 36, pp. 467-477, y el amplio estudio de Araceli López Serena, "La doble determinación del nivel histórico en el saber expresivo. Hacia una nueva delimitación del concepto de tradición discursiva", $R J, 62.3$ (2012), 59-97. 
con otro punto de vista, nacido de mi propia experiencia de análisis de los fenómenos verbales.

En su clásico Sincronía, diacronía e historia ${ }^{4}$, Eugenio Coseriu desarrolla con brillantez y profundidad los argumentos en contra de la interpretación predominante de la dicotomía saussureana entre sincronía y diacronía. Puesto que el objetivo central del Cours de linguistique générale de Saussure era fundamentar la investigación de las lenguas como sistemas, sus dicotomías de lengua y hablay sincronía y diacroní ${ }^{5}$ se orientaron a definir las condiciones de posibilidad del estudio lingüistico; sin embargo, las mismas afirmaciones de Saussure, como las interpretaron sus alumnos, se volvieron predominantes y establecieron una relación teórica esencial entre el sistema lingüístico, la lengua saussureana y la sincronía, que fue más allá de las condiciones epistemológicas de la investigación descriptiva a la suposición de que la naturaleza de la lengua es sincrónica, a partir de la noción de sistema que preconizaba Saussure; hay que señalar que esa noción era entonces, y aún ahora, más un desiderátum que una definición precisa del modo de organización de una lengua; sobre esa base el sistema, para muchos estructuralistas herederos de Saussure, debía ser inmutable; si no lo fuera, su coherencia interna estaría en cuestión. En consecuencia la evolución de una lengua, manifiesta en los cambios que haya sufrido a lo largo del tiempo, se convierte en mero accidente inexplicable. El mismo Saussure, como alumno que fue de la lingüística histórica alemana, propuso el concepto de diacronía como una proyección del devenir de la lengua, que se podría estudiar mediante una sucesión de estados sincrónicos; es decir, una sucesión como la de los cuadros de una película, que cuando pasan a cierta velocidad producen la ilusión del movimiento ${ }^{6}$.

Con una concepción de la naturaleza de la lengua de esa clase, el concepto de diacronía ha terminado por suplantar la historia, al grado

$42^{\text {a }}$ ed., Gredos, Madrid, 1973.

5 No considero la relación significado/significante como otra dicotomía; esta relación fundamenta la teoría del signo saussureana, sobre la que abundo en mi libro Ensayos de teoría semántica. Lengua natural y lenguaje científico, El Colegio de México, México, 2004.

${ }^{6}$ En su artículo "Vom Primat der Geschichte", Sprachwissenschaft, 5 (1980), p. 133, señala Coseriu: "El estudio sincrónico de la lengua sería en realidad el único adecuado, si la evolución lingüística tocara cada vez sólo elementos aislados de la lengua y tuvieran lugar fuera del sistema; [Saussure] ha mostrado sólo indirectamente, es decir, mediante una involuntaria reducción al absurdo, que es imposible integrar la evolución lingüística como un sistema lingüístico estático" ("Die synchronische Sprachbetrachtung wäre tatsächlich die einzig eingemessene, wenn der Sprachwandel jeweils nur vereinzelte Elemente der Sprache betreffen und außerhalb des Sprachsystems erfolgt; er hat nur indirekt, das hei $\beta t$, durch eine unfreiwillige reductio ad absurdum gezeigt, da $\beta$ es unmöglich ist, den Sprachwandel in ein als statisch aufgefaßtes Sprachsystem zu integrieren"). La traducción es mía. Este artículo de Coseriu resume, retomando, sus argumentos de Sincronía diacronía e historia y, naturalmente, añadiéndole sugerentes comentarios. 
de que muchos lingüistas creen que diacronía e historia son lo mismo. Bajo tales condiciones del pensamiento, la historia de la lengua, tal como se practicaba en el siglo XIX y mediados del Xx, por autores como Ramón Menéndez Pidal y Rafael Lapesa, o por Hermann Paul para la historia del alemán, quedó limitada a una filología cada vez más despreciada por la lingüística; lo que se entiende hoy predominantemente por historia de la lengua se ha convertido en una serie de cortes sincrónicos en los que el cambio y la evolución solamente pueden suponerse, sin establecer continuidades. En lo que Coseriu insiste es en que, con base en cortes sincrónicos para formar la diacronía, el cambio en cuanto tal es inexplicable.

No sólo eso: todo lo que haya podido condicionar o motivar ciertos cambios y todos los fenómenos sociales y culturales que hayan dado lugar a la evolución, pero que, obviamente, no forman parte del sistema lingüístico, quedan reducidos a accidentes anecdóticos, sin valor para el estudio diacrónico; se consideran objeto de una "lingüística externa", accidental y casi frívola; "lingüística blanda" de poco valor para la investigación.

La dicotomía saussureana de lengua y habla, como lo señaló primero Karl Bühler en su Teoría del lenguaje $e^{7}$ y después Coseriu en la obra citada, puede integrarse a una concepción más rica, definida por la distinción de Guillermo de Humboldt entre Werky Tätigkeit, que manifiestan en alemán la distinción aristotélica entre ergon y energeia, producto y actividad. Afirma Humboldt: "La lengua, considerada en su verdadera esencia, es algo continuo y a cada momento pasajero... No es un

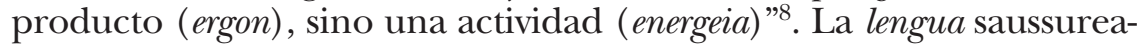
na corresponde al producto o ergon; el habla, en general, a la actividad, la energeia. Toda la lingüística descriptiva y la mayor parte de la teoría lingüística actual, junto con las disciplinas derivadas de ella, como la sociolingüística, se pueden explicar mediante la concepción saussureana y son lingüística del ergon.

Cuando se analiza la lengua como actividad y no como producto, las categorías de análisis de la lingüística descriptiva tienen que redefinirse, pues no son categorías elaboradas para analizar y comprender la actividad, sino sólo su producto, que es lo que se manifiesta en cada estado sincrónico en que se fijan los datos. Para situar el papel del ergon frente a la energeia, Bühler propuso su llamado "axioma C"9, a partir del cual la actividad verbal puede verse en su acción misma, es decir, en cuanto a la actuación de una persona hablando o, en un grado mayor

7 Sprachtheorie, Jena, 1934; en la edición ya citada de 1967, I, § 4.

8 "Die Sprache, in ihrem wirklichen Wesen aufgefasst, ist etwas beständig und in jedem Augenblicke Vorübergehendes... Sie selbst ist kein Werk (Ergon) sondern eine Thätigkeit (Energeia)", Wilhelm von Humboldt, VII, 46 (12). La traducción es mía.

9 Op. cit., § 4, pp. 93-126. 
de formalización, como acto verbal: como una acción dotada del sentido que una comunidad lingüística confiere a ciertas clases de acciones verbales que resultan inteligibles y valiosas para su actuación en el mundo. Reconocer la lengua como actividad implica reconocer el papel activo de los sujetos que la hablan, no abstraerlos y dejarlos de lado en el estudio de la lengua; los hablantes son sujetos de su propia lengua y en consecuencia sujetos de su propia historia. En este sentido, la actividad de los hablantes deja de ser una simple localización de la fuente de un dato, para poderse considerar en sí misma, en los hechos reales de sus acciones y sus actos verbales, que se producen siempre en una comunidad histórica. Con esta concepción, encontramos una manera de superar la epistemología del estructuralismo hacia una lingüística de la actividad verbal, una lingüística pragmática ${ }^{10}$, una lingüística de la evolución, una lingüística plenamente histórica.

Coseriu, en la misma obra antes citada y a partir del pensamiento aristotélico, avanza en la reflexión acerca de lo que es la actividad ver-

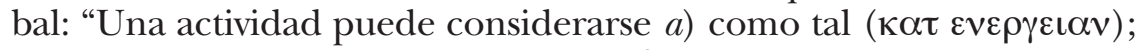
b) como actividad en potencia ( $\alpha \alpha \tau \alpha \delta v v \alpha \mu \nu$ ) ; y $c$ ) como actividad realizada en sus productos ( $\kappa \alpha \tau \varepsilon \rho \gamma o v)$ ". Según propone, la actividad verbal como tal, o hablar, "es una actividad universal que se realiza por individuos particulares, en cuanto miembros de comunida-

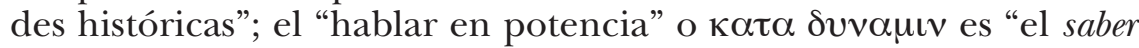
hablar, en el cual pueden distinguirse un escalón universal, otro particular, y otro histórico: este último es, precisamente, la «lengua» como acervo idiomático, o sea, como saber hablar según la tradición de una comunidad" tos ( $\kappa \alpha \tau \varepsilon \rho \gamma o v)$ es la lengua que encontramos en las grabaciones o en los textos que manifiestan un discurso temporal, circunstancial y socialmente determinado. Son estos productos los que estudia la lingüística digamos "canónica", sea de raigambre saussureana o de origen estadou-

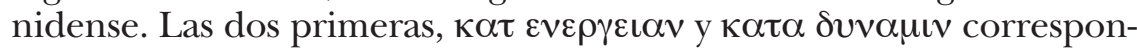
den a la energeia y, en consecuencia, a una lingüística de la actividad.

Si ahora introducimos la distinción bühleriana entre acción y acto verbal, se podrá considerar que la acción verbal de cada individuo en cada

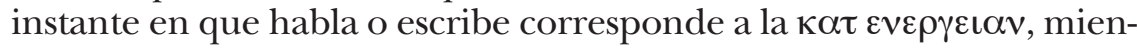
tras que los actos verbales, cuya pertinencia, cuya inteligibilidad, proviene del reconocimiento comunitario de que tienen sentido, correspon-

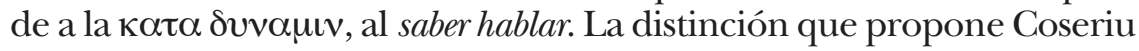

10 Hoy día hay varias concepciones de la "lingüística pragmática". Sigo los fundamentos que derivan de Wittgenstein, que se pueden resumir en la idea de que las expresiones verbales no se aplican a la manifestación de un sentido previo, sino que éste se forma en la actividad verbal; se elimina la cuestión ontológica de si para entender el significado de $\mathrm{X}$ hace falta que exista previamente un ente $\mathrm{Y}$ al que refiere; por el contrario, "el significado de X es su uso".

11 II, § 2.1., p. 45 . 


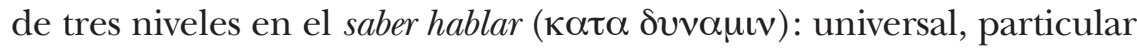
e histórico, puede reducirse a dos, pues el saber hablar universal no logra distinguirse del mero hablar como actividad ( $\alpha \tau \varepsilon \varepsilon v \varepsilon \rho \gamma \varepsilon \iota \alpha \nu$ ), sobre todo si se introduce la nota de "individuos particulares, en cuanto miembros de comunidades históricas". Es cierto: la experiencia indica que no hay un mero hablar, que se produzca fuera de una comunidad de habla, que es por definición histórica ${ }^{12}$; prueba de ello dan los estudios -hoy olvidados o escondidos- de "niños lobo" o "niños salvajes", de niños sustraídos a todo contacto social, que no aprenden a hablar, es decir, no ejercen su facultad innata del lenguaje y, en consecuencia, o mueren o alcanzan un nivel ínfimo de desarrollo de su inteligencia y de la lengua de su comunidad ${ }^{13}$. Si hubiera un mero hablar, universal por corresponder a lo humano, los niños lobo hablarían; se comprobaría la idea que dio origen a la leyenda de aquel déspota oriental que privó a un niño de todo contacto social, esperando a que, cuando hablara, surgiera la lengua primigenia. Más bien, el hablar como actividad cubre

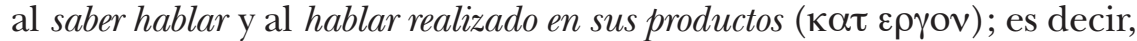
no se trata de una tripartición de la energeia, sino de una bipartición. El "hablar" como tal, fenómeno universal, no existe; todo hablar tiene una condición histórica, pues proviene de una tradición del hablar, gestada y transmitida por la sociedad; lo universal es la historicidad de las lenguas. Otra cosa es la facultad del lenguaje, hecho biológico que antecede a la posibilidad de hablar; pero en cuanto hecho biológico universal está más allá del saber hablary, como se ve en los casos mencionados de niños lobo, la facultad no se concreta por sí misma.

Esta modificación al pensamiento de Coseriu no altera el valor de su teoría, y permite continuar con otro elemento central de la concepción histórica de las lenguas. Dice Coseriu en su artículo de 1980 "Vom Primat der Geschichte": "Puesto que la lengua es una actividad creadora, el cambio no debe entenderse de manera solamente 'retrospectiva', en relación con la lengua previamente existente y como algo que sucede en y con esa lengua, sino a la vez y en primer lugar 'prospectivamente'. Desde este punto de vista el cambio no consiste en una sustitución en una lengua ya dada, sino en producción de lengua, objetivación histórica de lo creado en el habla, es decir, lengua sin más, en el momento de su evolución" ${ }^{14}$. En esta idea se reconoce la influencia de Humboldt

12 Un mero hablar sin considerar que se habla a otro, es característico de todas las concepciones solipsistas del lenguaje, como las de Searle y Chomsky.

${ }^{13}$ El último que, hasta donde llega mi información, ha destacado el papel de los estudios sobre "niños lobo", fue Erik LenNEBERG, en su libro Fundamentos biológicos del lenguaje, Alianza Editorial, Madrid, 1975.

14 "Da die Sprache eine schöpferischeTätigkeit ist, darf auch der Sprachwandel nicht nur «retrospektiv», mit Bezug auf die ihm vorhergehende Sprache und als etwas, was in und mit dieser Sprache geschieht, sondern er mu $\beta$ zugleich und an erster Stelle «prospektiv» verstanden werden. Und in dieser Hinsicht ist er nicht 
sobre Coseriu: "No se debe considerar la lengua como un producto ya muerto, sino más bien como una producción, y se debe abstraer más de ella que la mera designación de objetos y la manifestación del entendimiento; por el contrario, hay que volver cuidadosamente a la influencia mutua entre la actividad del espíritu y su origen"15.

Con estos conceptos puede uno concebir la historia de la lengua como un enorme complejo de fenómenos que se producen a lo largo del tiempo como fuente y resultado del saber hablar de la comunidad; es decir, como una tradición cuyas condiciones deben situarse con precisión en el estudio de todos los actos verbales que han dado lugar a ella. Había dicho antes que un acto verbal, siempre de naturaleza dialógica, adquiere su forma cuando la comunidad le reconoce un sentido y lo valora en cuanto tal. En consecuencia, la historia de la lengua consiste en el estudio de aquellos conjuntos de actos verbales que han ido dando lugar a la evolución de la lengua en condiciones históricas específicas.

El concepto de acto verbal así entendido da la clave para situar la actividad del hablante en relación con su comunidad. Esa actividad es, en primera instancia, acción verbal: el individuo dice algo para significar su experiencia de la vida; entre estas acciones hay muchas que son ante todo expresión de situaciones instantáneas, que disparan reacciones verbales de la misma clase; el mejor ejemplo es el de la interjección, que se manifiesta en situaciones de sorpresa, temor, exhorto, etc. Bühler considera esta clase de acciones verbales decires emprácticos. Estos decires no alcanzan a formalizarse en actos verbales mientras la comunidad no los valore como experiencias compartidas y los acuñe como expresiones de su lengua; sólo de esa manera interjecciones como ¡ay!, ¡caramba! u ¡órale! adquieren sentido. Otras acciones verbales tienen una finalidad comunicativa clara, son dirigidas por una póiesis, como lo explica Bühler. En esos casos, una vez que la comunidad lingüística las considera valiosas y pertinentes, formaliza el acto verbal con sus condiciones de posibilidad, pertinencia y sinceridad. Así, el acto verbal, que surge de la acción del hablante individual, se socializa y pasa a formar parte del acervo de posibles actos verbales que dan sentido a la comunicación social. Son el comienzo de la formación de las tradiciones de la lengua o tradiciones verbales. De esta manera pretendo responder una de las cuestiones que nos heredó Coseriu:

Ersetzung in einer schon gegebenen Sprache, sondern Entstehung von Sprache, historische Objektivierung des in der Rede Geschaffenen, das heißt, Sprache schlechthin im Moment ihres Werdens”, p. 135. La traducción es mía.

15 "Man muss die Sprache nicht sowohl wie ein todtes Erzeugtes, sondern weit mehr wie eine Erzeugung ansehen, mehr vor demjenigen abstrahieren, was sie als Bezeichnung der Gegenstände und Vermittlung des Verständnisses wirkt, und dagegen sorgfältiger auf ihren mit der innren Geistesthätigkeit eng verwebten Ursprung und ihren gegenseitigen Einfluss zurückgehen”, VII, 44 (11). La traducción es mía. 
"El verdadero problema del cambio lingüístico no es aquel del por qué del cambio en la lengua que lo precede (lo que debe ser así necesariamente), sino el del cómo de su aparición, es decir, de su constitución como tradición" 16 .

Ninguna lengua surge de repente. La más humilde, extraña y hasta primitiva de las lenguas humanas se ha venido formando en la sociedad que la habla y, a la vez, es la que ha venido dando forma a su sociedad. En los casos del español y de las lenguas que solemos conocer, su historia se remonta al indoeuropeo, al latín, al germánico o a un supuesto yutonahua, por ejemplo; de hablante en hablante, lo que tiene realidad concreta es lo que recibe de su comunidad y le permite entenderse, lo cual indica que todo individuo recibe un saber hablar de sus padres que ya constituye una tradición. Esa tradición se ha ido formando mediante un lento proceso de decantación del total de las acciones verbales de sus hablantes, logrado mediante sucesivas atribuciones de sentido, menudas las más veces, extraordinarias las menos, que dan lugar a actos verbales con los que se consolidan maneras de decir. A lo largo de la historia esas maneras de decir, que conviene llamar tradiciones verbales, van constituyendo un saber compartido que, en ciertos momentos, llevan a la reflexión: la sociedad reconoce como tales sus tradiciones verbales y las identifica como "su propia lengua"17; a partir de ese momento-que puede durar decenas o centenas de años- la noción de lengua se va estableciendo y permite que, al paso de la historia, se siga pensando en "la misma lengua", independientemente de los cambios que se hayan producido en sus tradiciones verbales. A partir de esta idea, resulta que lo que llamamos lengua a la manera saussureana es resultado de un doble proceso: por un lado, el conjunto de tradiciones verbales de una sociedad, descritas unitariamente; por el otro, la identificación social de esas tradiciones como parte o como pertenecientes a la misma lengua. Sólo tiene sentido hablar de cambio lingüístico y estudiarlo cuando existe una noción de lengua en la comunidad, construida sobre la realidad múltiple de sus tradiciones verbales.

En estas condiciones, la investigación de la historia de una lengua -la materia de la lingüística histórica- no puede reducirse a la yuxtaposición de estados sincrónicos en una diacronía, sino que supone el reconocimiento de procesos históricos de formación de las tradiciones verbales de la comunidad de habla, determinadas por la historia real de

16 "Und das eigentliche Problem des Sprachwandels ist nicht dasjenige des Warum der Veränderung in der ihm vorhergehenden Sprache (was er unbedingt sein mu $\beta$ ), sondern dasjenige des Wie seines Eintretens, das heißt, seiner Konstituierung als Tradition", "Vom Primat del Geschichte”, p. 135. La traducción es mía.

$17 \mathrm{Al}$ respecto considérense el ensayo de Tomás SEgovia, "De la misma lengua a la lengua misma”, en Miradas al lenguaje, El Colegio de México, México, 2007, y mi "Lengua histórica y normatividad", en el libro del mismo título, 2ª ed., El Colegio de México, México, 2009. 
los pueblos, de su vida política y cultural, de sus necesidades de expresión, de su contacto con otros pueblos.

Consideremos la historia del español ${ }^{18}$, que procede fundamentalmente del latín: los primeros documentos con que cuenta esa historia muestran un hablar latino, un hablar a la manera latina, un románice, que poco a poco va evolucionando: la distinción entre vocales largas y breves latinas se reinterpreta como distinción entre abiertas y cerradas; los casos latinos se reducen y cambia la estructura de la oración; las conjugaciones verbales se reducen y se mezclan; aumenta el uso de preposiciones. Ese romance sigue desarrollándose como tradición verbal de los hispanorromanos hasta que, a finales del siglo xII, tiene ya unas características que podemos concebir como el comienzo de una tradición hispánica. Para que los cambios del latín hacia el primitivo romance castellano se produjeran, eran necesarias ciertas circunstancias históricas de sus hablantes: la existencia de varios sustratos lingüísticos prerromanos en la Península ibérica, las características del latín hablado por las poblaciones hispanorromanas, el relajamiento de la comunicación con Roma, etc. Más tarde, por ejemplo, la acción creadora de los primeros frailes en La Rioja y Navarra, que se enfrentaron a la necesidad de atribuir nuevas letras a sus fonemas navarro-riojanos, pero a partir de otra tradición de escritura, enseñada por Alcuino de York en la época de Carlomagno ${ }^{19}$. Con la escritura, vuelta espejo de la pronunciación, en cuanto se les presentaba como una manera de reflexionar sobre sus propios fonemas (no como un medio automático de representar sus fonemas) se dio la primera identificación de las tradiciones verbales, aunque todavía no de una "lengua castellana", sino de un romance hispánico que más tarde contribuiría a la formación del castellano. Sólo cuando la actividad política de Alfonso el Sabio, durante el siglo XIII, requería del entendimiento entre todos los pueblos de su reino, esas tradiciones se reconocieron como "propia lengua", pero a la vez el rey dio comienzo a nuevas tradiciones verbales en la jurisprudencia, la historia y la ciencia, a partir de tradiciones verbales del árabe. Estas tradiciones eran, estrictamente hablando, tradiciones discursivas, es decir, tradiciones en que se había valorado un discurso orientado por una póiesis. Se puede establecer, en consecuencia, una distinción entre la tradición verbaly un importante elemento de ésta, que es la tradición discursiva. Si la tradición verbal nace de la valoración de actos verbales de la propia comunidad, la tradición discursiva, como lo demuestra la historia, se puede importar, se puede aprender de otras comunidades

18 Como lo muestran las obras dedicadas a la historia del español, como la Historia de la lengua española de Rafael Lapesa (hay muchas ediciones), aunque no la explican de esta manera. Véase mi Historia mínima de la lengua española, El Colegio de México, México, 2013.

19 Cf. Roger Wright, Latín tardío y romance temprano, Gredos, Madrid, 1989, pp. 201-202 y 307. 
lingüísticas. Sólo se puede comprender la historia de una lengua por esa clase de contingencias, que son del orden social y cultural, por lo que su estudio forma parte legítima de la lingüística histórica.

De manera que la evolución de una lengua no es un fenómeno natural, sino un fenómeno de la sociedad y de la cultura. Es imposible desentrañar un cambio, por ejemplo, la pérdida del plural latino en la formación del castellano si no se toma en cuenta, por supuesto, la pérdida de los morfemas de caso latinos, su reducción a dos casos oblicuos y el posible reforzamiento que les ofrecía el plural de origen celtibérico en -as y -os ${ }^{20}$.

Si miramos las tradiciones discursivas galorromanas e italianas, que dieron lugar a la formación de los cantares de gesta y al soneto, en siglos diferentes, tendremos que llegar a la conclusión de que esas tradiciones contribuyeron a la formación de las tradiciones cultas castellanas en la poesía épica y en la poesía amorosa. Si se adaptaron esas tradiciones al castellano fue por acontecimientos históricos específicos: la llegada de juglares franceses a territorio español, junto con la de cruzados que iban en busca de la fortuna en la lucha contra los musulmanes; y la transmisión culta del soneto renacentista italiano se explica mediante el papel que el humanismo italiano adquirió entre los intelectuales castellanos, como Juan de Mena y el Marqués de Santillana.

La historia de la lengua así concebida conduce a una reinterpretación y revaloración de muchos momentos de la historia social y cultural de los pueblos, y ofrece una explicación de los motivos del cambio, que definen la evolución de una lengua. Esa debiera ser la tarea de una lingüística histórica.

Luis Fernando LaRa Ramos

El Colegio de México

Miembro de El Colegio Nacional

${ }^{20}$ Cf. Rafael Lapesa, Historia de la lengua española, §17.1. 